\title{
Calculation of Static and Dynamic Characteristics for Fuel Consumption Regulator in Aircraft Engines, Taking into Account the Hydrodynamic Force
}

\author{
Asgat G. Gimadiev ${ }^{*}, 1$, Nikolay I. Kluev ${ }^{2}$ and Dmitry M. Stadnik ${ }^{1}$ \\ ${ }^{1}$ Samara State Aerospace University, 443086, Samara, Russia \\ ${ }^{2}$ Samara State University, 443011, Samara, Russia
}

\begin{abstract}
: the description of hydrodynamic force calculation methodology on the differential fuel consumption (thrust) control valve in aircraft engines (AE) by the software package ANSYS. The dependences of hydrodynamic forces on the fluid flow rate are developed through the triangular windows and the position of the differential pressure valve plug. The static and dynamic characteristics of the draft controller were calculated in the software package MATLAB/SIMULINK, taking into account the hydrodynamic force on the differential valve for different diameters of a damping jet. The possibility of the draft controller developed model use is specified at the calculation of AE dynamic characteristics.
\end{abstract}

Keywords: AE, calculation, differential valve, fuel consumption controller, graphic dependences, hydrodynamic force, static and dynamic characteristics.

\section{INTRODUCTION}

The consumption regulator is one of the major components and the $\mathrm{AE}$ and, ultimately, $\mathrm{RN}$ operation depends on its accuracy and reliability $[1,2]$. Therefore, the careful study of the regulator dynamic characteristics and the evaluation of its dynamic characteristics sensitivity concerning the rejection of its parameters is necessary. The methods of regulator calculation parameters and the methods of their static and dynamic characteristics study are known [3]. However, its hydrodynamic forces are not taken into account and this fact reduces the accuracy of the parameters calculation and the regulator characteristics. This article eliminates this gap due to the calculation and accounting of the hydrodynamic forces using the software package ANSYS. The consumption regulator is installed in the fuel supply line in a two-component $\mathrm{AE}$ gas generator. The entire consumption of one of the fuel components, for example, an oxidant is supplied to the gas generator, where it is mixed with the fuel part from the fuel consumption regulator output. The higher the fuel consumption rate in the gas generator, the higher the temperature of the "oxidated" gas and, consequently, the higher fuel pump power and $\mathrm{AE}$ traction.

\section{CALCULATION METHODS}

At the development of regulator equations (see Fig. 1), a number of simplifying assumptions is accepted: the fuel bulk creep compliance and regulator walls are not considered because of their smallness; the hydraulic losses in the throttling elements are taken into account by the quasi- (a)

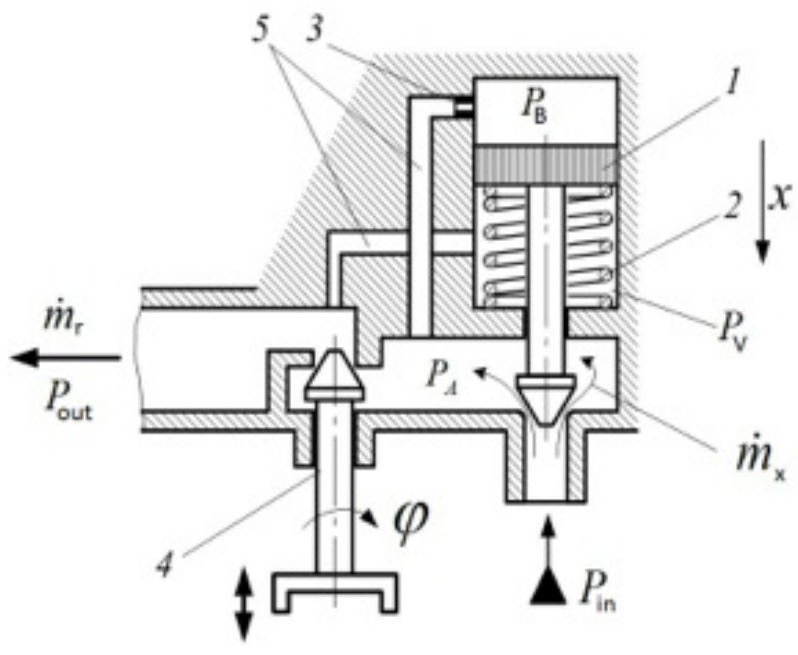

(b)

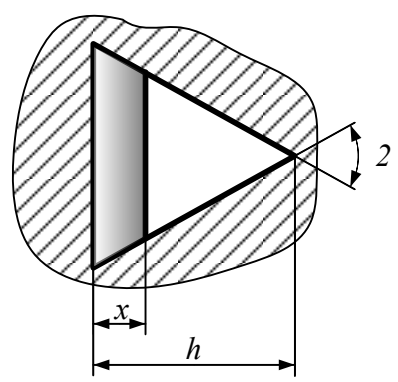

Fig. (1). Direct action flow regulator. (a) Schematic diagram of the hydromechanical regulator for direct action fuel component consumption: 1-differential valve; 2-spring; 3-jet; 4-adjustable throttle valve; 5 -ducts for pressure supply to the differential valve; (b) Triangular window diagram of the differential valve. 
stationary model; the dry friction in moving elements is absent; the fuel leakage in movable elements gaps of the differential pressure valve is small compared with the flow rate of the fluid through the jet; the hydraulic losses in the feedback channels are accounted by the laminar model of fluid flow; the hydraulic losses and the force of inertial resistance of regulator areas according to the main fluid flow are not considered because they are small compared to the losses of the throttle and the differential valve; the mass consumption of fluid through the narrow feedback channels is negligible compared to the fluid flow through the regulator; the throttling section of a differential valve is presented by four triangular windows (see Fig. 1b).

\subsection{Development of Flow Regulator Mathematical Model}

Taking into account the accepted assumptions the regulator algebraic and differential equations are derived. The solutions of such equations are the source of the regulator static and dynamic characteristics.

The equation of the differential valve motion is of particular interest.

$$
M_{p} \cdot \frac{d^{2} x}{d t^{2}}+\lambda_{\text {fric }} \cdot \frac{d x}{d t}+\gamma_{\text {spring }} \cdot \mathrm{x}=\mathrm{F}_{\mathrm{p}} \cdot\left(P_{\mathrm{B}}-P_{\mathrm{V}}\right)-N_{\text {spring }}+N_{\text {flow }}
$$

where $M_{p}$ is the reduced mass of the differential valve movable part, in kilograms; $x$-the valve coordinate along its axis, $\mathrm{m} ; t$-time, $\mathrm{s} ; \lambda_{\text {fric }}=\mu \cdot l \cdot b_{\mathrm{p}} / \delta_{\mathrm{p}}$-the coefficient which takes into account the viscous friction; $\mu$-the fluid dynamic viscosity, Pas; $l=\pi \cdot d_{\mathrm{p}}$-the length of the piston circumference, $\mathrm{m} ; d_{\mathrm{p}}$-piston diameter, $\mathrm{m} ; b_{\mathrm{p}}$-the ring surface width of the piston which is in contact with the plug, $\mathrm{m} ; \delta_{\mathrm{p}}$-the gap between the piston and the plug, $\mathrm{m} ; \gamma_{\text {spring }}$ the spring stiffness, $\mathrm{N} / \mathrm{m}$;-the piston area which is influenced by $P_{\mathrm{B}}$ and $P_{\mathrm{V}}$ pressures-the pressures in the $\mathrm{B}$ and $\mathrm{V}$ cavities, $\mathrm{m} 2 ; N_{\text {spring }}$-the spring preload (at x $\left.=0\right) \mathrm{H} ; N_{\text {flow }}$-the hydrodynamic force acting on the valve, $\mathrm{N}$.

The hydrodynamic force arises from the fact that during the fluid flow through the triangular windows the pressure in the narrow section falls by an amount proportional to the dynamic pressure:

$N_{\text {flow }}=c_{\text {flow }} \cdot \rho \cdot v^{2} \cdot F_{\text {win }} / 2$,

where $C_{\text {flow }}$ is hydrodynamic force coefficient; $\rho$-the liquid density, $\mathrm{kg} / \mathrm{m}^{3} ; v$-the fluid flow rate in the narrow section of throttling windows, m/sec; $F_{\text {win }}$-the equivalent area of the valve end face in the vicinity of the triangular windows, depending on the valve position, $\mathrm{m}^{2}$.

$c_{20}$ coefficient is similar to the coefficients introduced in fluid mechanics to determine the forces acting on a streamlined body. The liquid flow rate in the narrow section is determined by its volumetric flow rate at the regulator inlet.

$v=\dot{m}_{\mathrm{x}} /\left(\rho \cdot \mu_{x} \cdot F_{\mathrm{x}}\right)$, where in $\dot{m}_{\mathrm{x}}$ is the mass flow of fluid through the differential valve (at regulator inlet), $\mathrm{kg} / \mathrm{s} ; \mu_{x}, F_{\mathrm{x}}$-the flow coefficient and the cross sectional area of triangular windows, $\mathrm{m}^{2}$.

\subsection{Development of a Numerical Model for Flow Regulator with Ansys Software Package}

In a mathematical model of the regulator the hydrodynamic force arising in the course of the fuel expiration through the triangular window of the differential valve is weakly studied and it has a significant impact on the regulator static characteristics and its stability. Let's consider the calculation of the hydrodynamic force acting on the differential regulator valve. A geometric model of the regulator is developed for this purpose to meet the computational model requirements, on the basis of which its hydraulic domain in the CAD-module of Solid Works package is developed. In recent years, the software products based on the methods of computational fluid dynamics (CFD methods), supported by the development of numerical algorithms and the large capabilities of computers become widely used in aggregate elements to study the operational processes [4].

The set of equations that describes the momentum, heat and mass exchange is known as the Navier-Stokes equations. The analytical solution of the Navier-Stokes equations exist only for the simplest models of fluid flow under ideal conditions. A numerical approximation of equations using algebraic approximations, which can be solved with the help of CFD (Computational Fluid Dynamics) codes should be introduced to obtain the solution of real flow streams [5-10]. There are several numerical methods of solution which are used in CFD codes. The most common is the finite volume method.

The known commercial CFD codes solve the unsteady state Navier-Stokes equations: transportation; momentum conservation (momentum reservation equation); condition; thermal energy.

The conservation equations are quantized by the finite volume method, which make possible the discretization of a spatial domain using a grid [11]. The grid is used to develop the finite elements, which are in turn used to calculate the abovementioned variables such as mass, momentum, energy, etc., as all input variables and the fluid properties are kept in the nodes (grid points). In a general case, the grid may comprise tetrahedrons, prisms, pyramids and hexahedral elements. The control volume is stored between the grid points, using the average lines.

During the development of grid models several types of grids are used: structured, unstructured, and combined grids. The use of the structured hexahedral grids enables to obtain the high accuracy of the solution at a relatively low time costs for the calculation process. However, their development may be quite time-consuming process compared to the process of unstructured grids development. The unstructured grids do not always reduce the solution accuracy, but the calculation time is greatly increased compared with structured grids.

The use of combined grids calculation model allows you to combine the advantages of both grid types. At this 
approach, the calculation model is composed of both structured and unstructured grids.

The recommendations for grid model development of any of computational domain are as follows:

1) It is necessary to strive to ensure that the grid lines are perpendicular to the solid boundaries of a model. The element angles must be within the range from 200 to 1600 . Note that at the element angle values less than 400 the solution accuracy and its convergence are significantly reduced;

2) the availability of cells with negative volume is not admitted;

3) is the best option is the grid lines parallel to the intended direction of flow;

4) the changes of the grid cell sizes should be smooth. The optimal ratio of neighboring cell areas is the range of $0.75 \ldots 1.5$. The particular attention to this item should be paid in the places of high gradient flow rates (input and output element edges, in the gaps and near the walls, etc.).

The calculation of the first cell thickness should be performed in the following sequence:

\subsubsection{Reynolds Number Determination}

$\operatorname{Re}=\frac{\rho \cdot U \cdot D}{\mu}$

where $\mathrm{D}$ is characteristic dimension of the cross section within the different sections of the regulator flow part.

The internal friction coefficient determination in the boundary layer

$C_{f}=\frac{0,078}{\operatorname{Re}^{1 / 4}}$

Shear stress determination on the walls

$\tau_{w}=\frac{1}{2} C_{f} \rho \cdot U^{2}$

Determination of wall tangent velocity

$U_{\tau}=\sqrt{\frac{\tau_{w}}{\rho}}$

On the basis of the desired $y+$ value from the following equation

$y_{p}=\frac{y_{p}^{+} v}{U \tau}$

the thickness of the first equation layer is determined.

The fluid flow in aggregates is mainly turbulent one. The velocities in the above mentioned equations are the average ones, and therefore additional stress component terms are necessary in the momentum equation. These stresses are modeled using the turbulent viscosity, which is calculated using the turbulence models, for example $k-\varepsilon$, or Shear Stress Transport (SST) [11]. Although the exact prediction of the turbulent flow behavior is not possible, you may try to develop a numerical model that reproduces some of the turbulent motion statistical characteristics.

There are three main approaches to the numerical simulation of turbulence [12]:

- $\quad$ Direct Numerical Simulation (DNS);

- $\quad$ Large Eddy Simulation (LES);

- $\quad$ Reynolds averaged Navier-Stokes, (RANS).

The DNS method is the most reasonable and accurate one, but it requires a detailed spatial and temporal resolution and, therefore, leads to a large computational costs. The application of LES method is not always accurate and adequate for aggregate flow problem solution.

The turbulence models, used in engineering calculations, are usually based on the RANS turbulence models. This method implemented the statistical approach to the turbulence study. This approach is the averaging of the equation motion. All motion parameters are decomposed into high and turbulent components. The RANS averaging means the averaging over an ensemble of states. In the momentum equation in the form of the Navier-Stokes equation the Reynolds stresses appear which should be closed. The closure of the Reynolds equations (the turbulent stress determination) is performed by using semi-empirical turbulence models [13]. The following semi-empirical models are used:

- The models using the Boussinesq hypothesis (algebraic models);

- $\quad$ Single equation models (Spalart-Allmares SA model, Sekundov model);

Two equation model ( $k-\varepsilon$ type model);

$k-\omega$ type model;

- $\quad$ SST Menter model, based on $k-\omega$ model);

- $\quad$ Reynolds stress models (differential Reynolds stress model (DRSM));

- $\quad$ Algebraic Reynolds stress models (ARSM);

- $\quad$ Explicit algebraic Reynolds stress model (EARSM).

The most common approach to the turbulence modeling used in engineering problems is "RANS" approach. As a rule, $k-\varepsilon$ or SST (Menter model) is used. Let's give the description of the SST model using the automatic wall function for clarity. This model is based on two equations: for the kinetic turbulent energy $\mathrm{k}$ and its frequency $\omega$. At that the near-wall region uses $k-\omega$ turbulence model, the external flow uses $k-\varepsilon$ model. $\varepsilon$ is the dissipation of kinetic energy. The equation for the turbulent kinetic energy and its frequency determination:

$$
\begin{aligned}
& \frac{d k}{d t}=\nabla \cdot\left(\left(v+\sigma_{k} v_{T}\right) \nabla k\right)+P_{k}-\beta * \omega k ; \\
& \frac{d \omega}{d t}=\nabla \cdot\left(\left(v+\sigma_{\omega} v_{T}\right) \nabla \omega\right)+\frac{\gamma}{v_{T}} P_{k}-\beta \omega^{2}+ \\
& +\left(1-F_{1}\right) \frac{2 \sigma_{\omega 2}}{\omega}(\nabla k) \cdot(\nabla \omega),
\end{aligned}
$$


where $v_{T}=\frac{k}{\omega}$ is the turbulent viscosity; $P_{k}$ is the member, responsible for the generation of vortices; $F_{1}$ is the function of $k-\omega$ and $k-\varepsilon$ models alteration.

The constants forming the equation (9) and (10) are presented in Table 1 [11].

Table 1. Model coefficients for SST turbulence model.

\begin{tabular}{|c|c|c|c|}
\hline Parameter & Value & Parameter & Value \\
\hline \hline$\beta_{1}^{*}$ & 0,09 & $\beta_{2}^{*}$ & 0,09 \\
\hline$\beta_{1}$ & 0,055 & $\beta_{2}$ & 0,0928 \\
\hline$\alpha_{1}$ & 0,25 & $\gamma$ & $\frac{\beta}{\beta^{*}}-\frac{\sigma_{\omega} k^{2}}{\sqrt{\beta^{*}}}$ \\
\hline$\sigma_{k 1}$ & 0,85 & $\sigma_{k 2}$ & 1,0 \\
\hline$\sigma_{\omega 1}$ & 0,5 & $\sigma_{\omega 2}$ & 0,81 \\
\hline
\end{tabular}

The following assumptions are used as a rule at the hydrodynamic processes simulation [14]:

- The thermodynamic effect of cavitation is neglected;

- $\quad$ Mas sources arise from the phase transfer;

- $\quad$ Mixture phases fill the entire reviewed volume;

- The barotropic pressure dependence on the temperature is used;

- $\quad$ The Boussinesq hypothesis is used;

- The constant boundary conditions averaged over the entire surface are used during the stationary calculations;

The direction of fluid flow at the computational domain inlet and outlet is perpendicular to the surface;

- $\quad$ All the flow rate components are set to be zero foe the walls (condition of the working fluid adhesion).

The boundary conditions during the stationary calculations are selected on the basis of the actual operating modes of aggregates. It is necessary to carry out a series of numerical experiments for one of the unit real operating conditions of the unit to select the appropriate boundary conditions, as well as sustainable solutions. It is necessary to pay attention to the solution accuracy and the rate of its convergence. For example, the most appropriate boundary conditions for the flow regulator are the conditions under which the full input pressure and the output consumption is set.

\section{RESULTS}

In the process of the hydrodynamic parameters calculations the velocity and the pressure of the liquid stream is determined. By these parameters integration the fluid flow, the average pressure at the outlet of the differential valve windows is determined. By integrating the pressure distribution of the liquid on the plug surface of th differential valve the axial component of the required hydrodynamic force is calculated.

The example of velocities calculation is shown by Fig. (2).

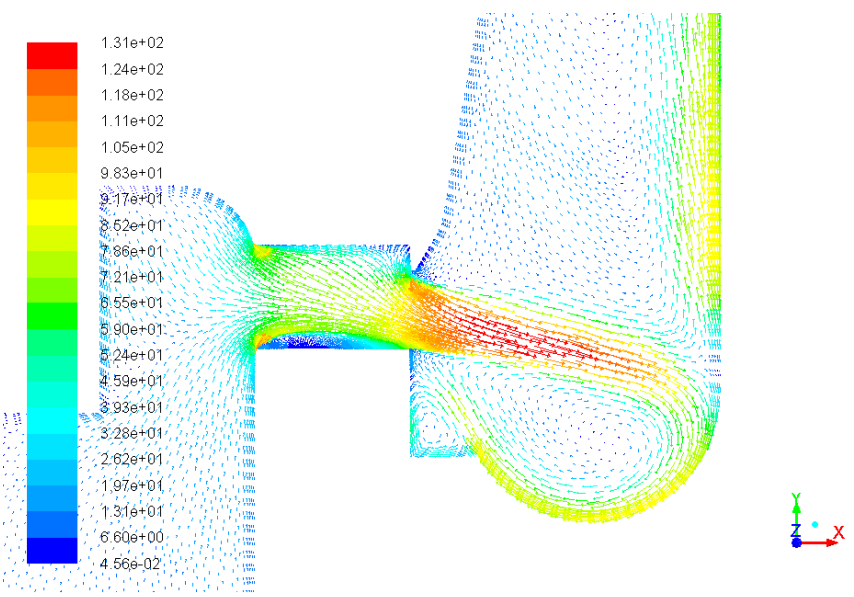

Fig. (2). Streamlines speeds during the fluid motion through the triangular windows of the differential flow regulator valve, $\mathrm{m} / \mathrm{s}$.

The hydrodynamic force on the differential valve depends on the fluid flow rate through the triangular windows and the valve edge position relative to the windows. The greater the velocity of fluid flow and the flow area of triangular windows, the greater the hydrodynamic force is $[15,16]$. In order to develop such a dependence we performed additional calculations in the ANSYS package and created the corresponding dependence in the form of a three coordinate grid surface (see Fig. 3).

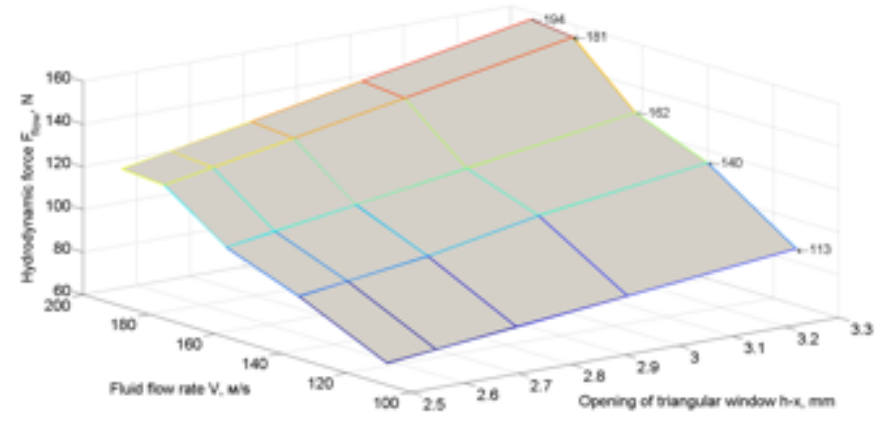

Fig. (3). Dependence of the hydrodynamic force on the differential flow regulator valve on the fluid velocity through the triangular windows and the differential valve turn.

The approximating dependence $N_{\text {flow }}=f(v, x)$ allows to take into account the hydrodynamic force at the calculation of the flow regulator static and dynamic characteristics, which are calculated by the use of the above equations with numerical methods in MATLAB/SIMULINK software package.

The method of the hydrodynamic force calculation developed by the authors is the basis for optimization problems solution by choosing the shape and size of differential valve windows, which satisfy the requirements of its static characteristics provision.

The transient and frequency characteristics of the flow regulator are calculated by the control and disturbing effects. The control effect is the throttle crane opening area, and the 
disturbance effect is the pressure change at its input and different diameters of the damping jet (see. Fig. 1).

This paper analyzed the influence of the jet diameter on the transient characteristics of the flow regulator. The jet diameter reduction from 2.8 to $0.8 \mathrm{~mm}$ resulted in parameters oscillation decrease of the flow regulator, while the opening differential pressure valve was increased by 0.06 sec. at the case stepwise increase of the working fluid pressure from 12 to $15 \mathrm{MPa}$.

At the increase of the throttle crane opening area from 240 to $265 \mathrm{~mm}$ the diameter impact on the opening speed of the differential valve affected to a lesser degree.

The frequency characteristics of the flow regulator are used to evaluate the stability of the "motor-regulator". One of the regulator parameters used to sustain the said system is the hydraulic resistance or the jet diameter. The results of amplitude-frequency characteristics of the draft regulator study determined that the regulator operates like a typical oscillating ATS unit with little resonance at the frequency of $54 \mathrm{~Hz}$ at the jet diameter of $2.8 \mathrm{~mm}$ and it operates as an aperiodic model unit of the second order at the jet diameter of $0.8 \mathrm{~mm}$.

\section{CONCLUSION}

The developed mathematical model of the flow regulator may be used to explore the $\mathrm{AE}$ dynamic characteristics, to predict the deviations of its characteristics due to process parameter variation of the unit elements or because of some defect in their design.

This work was supported by the Ministry of education and science of the Russian Federation in the framework of the implementation of the Program of increasing the competitiveness of SSAU among the world's leading scientific and educational centers for 2013-2020 years.

\section{CONFLICT OF INTEREST}

The authors confirm that this article content has no conflict of interest.

\section{ACKNOWLEDGEMENTS}

Declared none.

\section{REFERENCES}

[1] A.A. Goryachkin, A.E. Zhukovsky, S.M. Ignachkov, and V.P. Shorin, "Flow regulators for fuel systems of aircraft engines", Mechanical Engineering, pp. 200, 2000.

[2] A.G. Gimadiyev, "Choice of parameters, the calculation of the static and dynamic characteristics for fuel regulator", Samara: State Aerospace University publication, pp. 63, 2007.

[3] E.V. Lebedinsky et al., "Computer models of liquid rocket engines", Mechanical Engineering, pp. 376, 2009.

[4] V. Sverbilov, D. Stadnik, and G. Makaryants, "Study on dynamic behavior of a gas pressure relief valve for a big flow rate", ASME / BATH 2013 Symposium on fluid power and motion control, FPMC 2013.

[5] B.V. Ovsyannikov, and B.I. Borovsky, "Theory and calculation of supply aggregates for liquid rocket engines", [Tutorial for aircraft universities], Mechanical Engineering, pp. 375, 1986.

[6] A.I. Babkin, S.I. Belov, and N.B. Rutovsky et al., "The theory of automatic control with rocket propulsion systems", Machinery, pp. 328, 1978.

[7] A.A. Sheviakov, V.M. Kalnin, N.V. Naumenkova, and V.V. Dyatlov, "Theory of rocket engine automatic control", Mechanical Engineering, pp. 320, 1987.

[8] B.F. Glickman, "Automatic control of liquid rocket engines", Mechanical Engineering, pp. 296,1989.

[9] CFX-TASCflow manuals, version 15 [Text]. - AEA Technology, 2010.

[10] FIDAP manuals version 8.52. [Text]. - FLUENT Incorporated, 2009.

[11] Page, M. Turbulent Flow Computations in Turbine 99 Draft Tube with CFX-TASCflow, FIDAP and FINE / Turbo Turbine 99 [Text]

/ M. Page, A. Giroux // The second ERCOFTAC Workshop on Draft Tube Flow: workshop 2 - Älvkarleby, Vattenfall Utveckling AB, Sweden, 2001.

[12] A.Y. Snegirev, "High-performance computations in technical physics. Numerical simulation of turbulent flows", Tutorial - St. Petersburg: Polytechnical University publication, pp. 143, 2009.

[13] J. Taekyu, "Static characteristics of a flow regulator for a liquid rocket engine", Journal of Spacecraft and Rockets, vol. 48, pp. 541-4, 2011.

[14] L. JunHai, Y. NanJia, Z. Peng, and C. GuoBiao, "Design and integrated simulation of a pressurized feed system of the dualthrust hybrid rocket motor" School of Astronautics, Beihang University, Beijing 100191, China April 2013 vol. 56, 989-1000, doi: 10.1007 / s11431-013-5156-y.

[15] Ring, Elliot, "Rocket Propellant and Pressurization Systems", Prentice Hall, 1964.

[16] E.V. Lebedinsky, "Multifunctional Mathematical Simulation of Liquid Rocket Engines (Principles of System Synthesis)", Conference on Propulsive Flows in Space Transportation Systems. Bordeaux, France, 11-15 September 1995, pp. 514-28. 